\title{
A visualized exploration in urban sensing of air quality with vehicular sensors
}

Rita T. Tse and Philip Iat Seng Lei, Chon Tou Lam and Giovanni Pau

Computing Program, Macao Polytechnic Institute, Rua de Luis Gonzaga Gomes, Macao UCLA Computer Science Department, Los Angeles, CA, USA

University Pierre et Marie Curie, Paris, France

E-mail: ritatse@ipm.edu.mo,philiplei@ipm.edu.mo,p1204463@ipm.edu.mo, gpau@cs.ucla.edu

www.ipm.edu.mo

\begin{abstract}
Worldwide deterioration of air quality has raised people's concerns on what they inhale on a daily basis. This work exploits inexpensive pollution sensors deployed in the Macao Polytechnic Institute service fleet as a mean to monitor air quality at city scale. We propose web-based geo-spatial exploration tool to provide air-quality information for users and insights on the causes to the city hall. We are able to identify strong connections between the territory orography and the air quality level. Specifically, it is possible by analyzing various areas to discover that places with traffic congestion and confined space, or poor natural ventilation which exhibits a sudden change in air quality readings.
\end{abstract}

Keywords: Air Quality; Smart City; Mobile Sensing

\section{Introduction}

Air quality has been deteriorating in this few decades due to various factors, including factory wastes, combustion of petroleum and radioactive fallout. It has become important to monitor the air quality of different places. The MPI urban sensing research project [1][2] provides a cost-effective method to monitor air quality in Macao. It uses the campus vehicular testbed based on MPI facility vehicles to collect pollution data from many mobile air quality sensors.

To evaluate the air quality in Macao, we have developed an interactive interface to explore the sensor data in vehicle traces, and perform a pilot study of how air quality fluctuates in different situations.

This paper is organized as follows. We first compare our approach of air quality monitoring using sensor data in vehicle traces with the conventional approach of static monitoring stations. Then, we verified the correctness of the sensor data in the dataset. Next, we used the web-based exploration tool we 
developed to visualize the sensor data in vehicle traces, and highlight some observation in our pilot study of the data. We conclude this paper with some plausible improvements to current design.

\section{Related Work}

Data collected from the MPI urban sensing research project are used in this project to conduct interactive exploration and air quality analysis. Collection of data with MPI vehicular testbed can spread throughout Macao for the entire vehicle course with possible errors and deviations. Besides, a number of limitations are associated with this approach. For example, GPS receivers depend on at least 4 direct contact of GPS satellites in order to trilaterate user location, and due to tightly packed, skyscraper filled urban areas in Macao, in some occasions, the vehicle may fail to obtain an accurate GPS signal. To tackle the problem, procedures to evaluate the validity of raw data from GPS sensors is strictly required.

Tab. 1 Limitations on vehicular sensor exploration vs. conventional monitoring stations

\begin{tabular}{|c|c|c|}
\hline 1 & Vehicular Sensor Exploration & Conventional Monitoring Station \\
\hline 2 & $\begin{array}{c}\text { A number of factors contribute to } \\
\text { the inaccurate GPS readings for } \\
\text { determining location. }\end{array}$ & $\begin{array}{c}\text { Location determination via GPS does } \\
\text { not apply to static monitoring stations. }\end{array}$ \\
\hline 3 & $\begin{array}{c}\text { Air quality sensors reading may not } \\
\text { be as accurate as static monitoring } \\
\text { stations. }\end{array}$ & $\begin{array}{c}\text { Higher data accuracy is to be yielded } \\
\text { with more sophisticated apparatus. }\end{array}$ \\
\hline $\begin{array}{c}\text { sensors data in close proximity of } \\
\text { the vehicle course. }\end{array}$ & $\begin{array}{c}\text { Conventional approach only includes } \\
\text { sensors data for the proximity of } \\
\text { several monitoring spots. }\end{array}$ \\
\hline
\end{tabular}

Tab. 1 lists the limitations for vehicular sensor exploration compared to conventional monitoring stations on the data sampling aspect. Firstly, the accuracy of GPS readings depends on three major factors for vehicular sensor exploration, namely quality of the GPS receiver, satellites' position in relation to user position, and the characteristics of the environment. On the contrary, due to the semi-permanent setup of conventional monitoring stations, location determination by GPS is not required. Secondly, sensors are sophisticated equipment. Presumably, it is possible for the vehicle or environment to introduce interference to sensors. For example, vehicle operation induced vibrations can cause less than optimal measurements. On the other hand, a controlled environment is provided at monitoring stations. Lastly, another key advantage of 
conventional monitoring stations on data sampling is the constant and uninterrupted operation around-the-clock.

The exploratory dataset from MPI urban sensing research project will not fit in the conventional visualization approach [3][4]. The first reason is that the sensor data samples are split into intermittent routes in vehicle traces. The second reason is that sensor readings are taken in different places. Therefore we need to design and develop a new visualization method to show the change of sensor readings while the car is moving around in the city.

\section{Data Cleansing and Verification}

The dataset obtained are scattered and lacks aggregation. After removing sensor types with dubious data values, we chose five sensor types, namely "Pressure mbar", "\%RH”, “TOLUENE ppm", "ETHANOL ppm” and "H2S ppm” remain. We then verified that the sensor readings fall into reasonable range. Tab. 2 provides a detailed explanation.

Tab. 2 Accepted Data Types

\begin{tabular}{|c|c|c|}
\hline Entry & Quantity & Descriptions \\
\hline Pressure mbar & 75905 & $\begin{array}{r}\text { Entries range from 973 to 1051. Readings spread is close } \\
\text { to, or near the standard atmospheric pressure, } \\
1013.25 \text { mbar. }\end{array}$ \\
\hline TOLUENE ppm & 75767 & $\begin{array}{r}\text { Entries range from 0 to 439, according to [5][6]. Toluene } \\
\text { concentration within this range is acceptable. }\end{array}$ \\
\hline ETHANOL ppm & 75736 & $\begin{array}{r}\text { Entries range from 0 to 3798, according to [7][8][9][10]. } \\
\text { The highest value for ethanol concentration is fairly } \\
\text { acceptable. }\end{array}$ \\
\hline H2S ppm & 75738 & $\begin{array}{c}\text { Entries range from 0 to 5481, no negative values for this } \\
\text { entry was recorded. }\end{array}$ \\
\hline GPS & $\sim 3.8 \mathrm{M}$ & $\begin{array}{r}\text { Geospatial location. Deviate entries that are far away from } \\
\text { Macau were removed. }\end{array}$ \\
\hline
\end{tabular}

\section{Results and Discussion}

We have developed a web-based exploration tool for exploring the air quality sensor data collected by MPI urban sensing research project and study factors that may contribute to the deterioration of air quality. Fig. 1 is the interactive exploration part of the web application. 


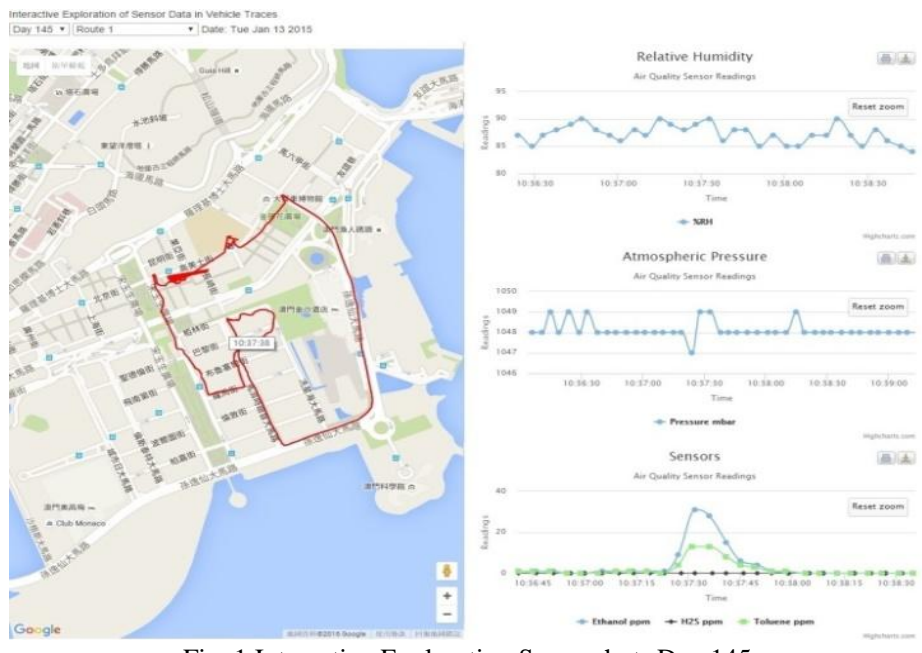

Fig. 1 Interactive Exploration Screenshot -Day 145

At the left-upper corner, two drop-down menus are provided for a user to specify a day. After a day is chosen, the application will be able to dynamically break vehicle trace data in the day into separate route information. Afterwards, the user can select a route. The system will then process the inputs by the user (selected day and route) and give multiple outputs, which includes GPS coordinates data and air quality sensors data. On the interface, the top portion provides a space to display the full date of the selected day to clarify when the event happened. On the left side of the interface, a map is employed to render vehicle trace. Lastly, on the right side of the interface, several charts present sensors data; from top to bottom, the first two charts display generic environmental information, the last chart combines three crucial air quality sensors measurements.

\section{Observation and Discussion}

We used the exploration tool to inspect situations of spikes of sensor readings in a pilot study. We observed four common cases: 61)

-Traffic congestion in heavy traffic zones, especially in rush hour (e.g. Day

- Poorly ventilated areas (E.g. in Day 91, when the vehicle traveled through a tunnel)

- Bus stops nearby (E.g. in Day 145 in the car trace shown in Figure 1, there were multiple bus stops) 
-Steep uphill climb for vehicles (E.g. in Day 147). The spike may be caused by a vehicle from that uphill climb produced excessive harmful substances from gasoline combustion, and was caught by the urban sensing vehicle from behind.

Fig. 2 illustrates a common trait of these four cases. There were simultaneous increase of ethanol and toluene. Both ethanol and toluene are ingredients of gasoline. For this reason, it is believed to some extent, that gasoline combustion plays an important role to the observed results.

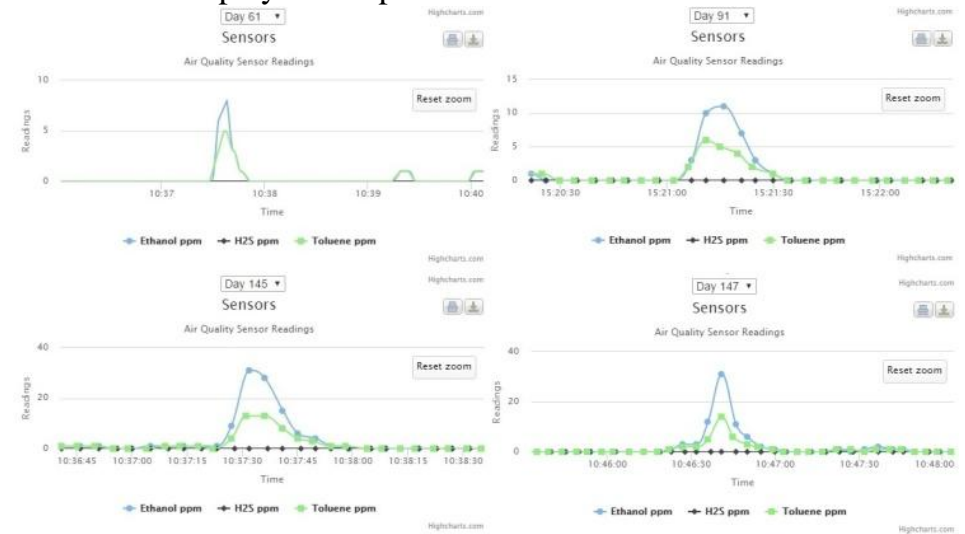

Fig. 2 Interactive Exploration Screenshot - Common Trait

\section{Conclusion}

This study makes use of the data collected from the MPI urban sensing research project to inspect air quality around Macao. In the end, this study. has yielded a web-based application which allows users to conveniently inspect and discover air quality throughout Macao. In addition, users can potentially investigate different situations that affect air quality by co-relating the vehicle traces and the air quality sensor data.

A web-based application has opened the door to further functionalities expansion. It is possible to develop more visualization tools, such as heatmap and scatter plot into the web interface to further improve and perfect the current design.

\section{Acknowledgments}

This project is supported by Macao Polytechnic Project N. (RP/ESAP-02/2014)

- Bridging Urban Sensing and Social Networks. 


\section{References}

1. Pau, G., \& Tse, R. Challenges and opportunities in immersive vehicular sensing: Lessons from urban deployments. Signal Processing: Image Communication, 900-908.

2. Lutterotti, P., Pau, G., Jiang, D., Gerla, M., \& Delgrossi, L. C-VeT, the UCLA Vehicular Testbed: An Open Platform for Vehicular Networking and Urban Sensing.

3. SMG. Air Quality Index Bulletin. http://www.smg.gov.mo/www/ccaa/iqa/daily/fe_iqa_index_latest.htm [Oct 13, 2015].

4. SMG. Concentration of Air pollutants. http://www.smg.gov.mo/smg/airQuality/e_air_conc.htm [Oct 13, 2015]

5. National Center for Biotechnology Information. PubChem Compound Database. Compound Summary for CID 1140: Toluene.

6. http://pubchem.ncbi.nlm.nih.gov/compound/toluene\#section=Top, 2004 [Sept 21, 2015].

7. CDC. International Chemical Safety Cards(ICSC): Toluene. http://www.cdc.gov/niosh/ipcsneng/neng0078.html, 1994 [Sept 21, 2015].

8. National Center for Biotechnology Information. PubChem Compound Database. Compound Summary for CID 702: Ethanol. http://pubchem.ncbi.nlm.nih.gov/compound/702\#section=Top, 2004 [Sept 21, 2015].

9. Brust, J. C. M.. Ethanol and Cognition: Indirect Effects, Neurotoxicity and Neuroprotection: A Review. International Journal of Environmental Research and Public Health, 7(4), 1540, 1557. http://doi.org/10.3390/ijerph7041540, 2010.

10. Clean Air Trust. Sources and Availability of E85. http://www.cleanairtrust.org/Sources-Availability-E85.html, 2008 [Sept 22, 2015].

11. CDC. International Chemical Safety Cards(ICSC): Ethanol (Anhydrous). http://www.cdc.gov/niosh/ipcsneng/neng0044.html, 1994 [Sept 22, 2015]. 